\title{
ROVING PEN
}

\author{
Manish Hinduja ${ }^{1}$ (D)
}

Received: 12 January 2021 / Accepted: 15 January 2021 / Published online: 3 February 2021

(C) Indian Association of Cardiovascular-Thoracic Surgeons 2021

In this evolving world of scientific innovations, it is prudent to stay updated with the current research work globally. This is a summary and reflection of important articles published recently in the various journals of cardiothoracic and vascular surgery across the world.

I. Association of radial artery graft vs saphenous vein graft with long-term cardiovascular outcomes among patients undergoing coronary artery bypass grafting: a systematic review and meta-analysis-by the RADIAL group

Gaudino M, Benedetto U, Fremes S, et al. RADIAL Investigators. Association of radial artery graft vs saphenous vein graft with long-term cardiovascular outcomes among patients undergoing coronary artery bypass grafting: a systematic review and meta-analysis. JAMA. 2020;324:179-187. doi: https://doi.org/10.1001/jama.2020.8228.

Will the radial artery (RA) beat saphenous vein grafts (SVGs) 10 years after coronary artery bypass grafting $(\mathrm{CABG})$ ?

\section{Key points}

- This study represents the best available long-term data on the potential value of using the RA as a bypass conduit.

- It is a patient-level pooled analysis comparing RA vs SVG in adult patients undergoing isolated CABG from 5 countries (Australia, Italy, Serbia, South Korea, and the UK) with enrollment from 1997 to 2009 and follow-up completed in 2019. Patients were randomized to undergo either RA $(n=534)$ or saphenous vein $(n=502)$ grafts for CABG.

Manish Hinduja

drmanishhinduja@gmail.com

1 Consultant Cardiothoracic and Vascular Surgery, Fortis Hospitals, Mumbai, India
- At a median follow-up of 10 years, the use of the $R A$, compared with the $S V G$, was associated with a statistically significant reduction in the incidence of the composite outcome of death, myocardial infarction (MI), or repeat revascularization and of the composite of death or MI.

Comments To date, several randomized controlled trials (RCTs) have compared SVG to RA use in CABG. While most trials have shown improved patency of the RA, none of them has been able to demonstrate a survival benefit using the arterial conduit. However, none of the existing RCTs was appropriately powered to detect a difference in clinical outcome. With this framework in place, the beginnings of the Radial Artery Database International Alliance (RADIAL) project were formed. The present study by Gaudino et al. extends the previous meta-analysis (of 5 years follow-up) to 10 years of follow-up and provides additional important insight. The longer follow-up is critical, as failure of SVGs typically occur between 5 and 10 years after CABG. Analyses of the components of the composite outcome were not prespecified. Accordingly, the reported reduction in mortality is considered a post hoc finding and hypothesis generating, but not definitive. One of the major strengths of the study is that it used patient-level, rather than study-level, data.

Take-home message If physicians and patients chose CABG, internal thoracic artery (ITA) graft to the left anterior descending coronary artery is essential. While awaiting further confirmatory evidence from long-term outcome trials, for use of additional conduits, if feasible radial arteries, should be given a preference over veins.

II. Prediction of mortality rate in acute type A dissection: the German Registry for Acute Type A Aortic Dissection score

Czerny M, Siepe M, Beyersdorf F, et al. Prediction of mortality rate in acute type A dissection: the German Registry for Acute Type A Aortic Dissection score. Eur J Cardiothorac 
Surg. 2020;58:700-706. doi: https://doi.org/10.1093/ejcts/ ezaa156.

\section{Key points}

- Data of 2537 patients enrolled in German Registry for Acute Type A Aortic Dissection (GERAADA) was used to develop a scoring system in the form of a Web-based application for predicting the 30-day mortality rate after type A aortic dissection (TAAD) repair.

- The Web application based on the final model can be found at https://www.dgthg.de/de/GERAADA_Score.

- Score is based on the following: age, need for catecholamines at referral, preoperative resuscitation, need for intubation before surgery, preoperative hemiparesis, coronary mal-perfusion, visceral mal-perfusion, dissection extension to the descending aorta and previous cardiac surgery.

Comments TAAD surgery is associated with many complications and unpredictable outcome. However, many risk factors contribute to its outcome. Whereas risk prediction models and scores are available for several other acute and chronic underlying diseases requiring open-heart surgery, no score for acute type A aortic dissection is available to date.

Take-home message The GERAADA score is a simple, effective tool to predict the 30-day mortality rate for patients undergoing surgery for acute TAAD.

III. An evaluation of the long-term patency of the aortocoronary bypass graft anastomosed to a vascular prosthesis

Ai K, Daisuke Y, Koichi T, et al. For Osaka Cardiovascular Surgery Research Group (OSCAR). An evaluation of the long-term patency of the aortocoronary bypass graft anastomosed to a vascular prosthesis, Eur J Cardiothorac Surg. 2020;58:832-838, https://doi.org/10.1093/ejcts/ezaa179.

\section{Key points}

- Retrospective study wherein 84 patients with concomitant CABG who underwent surgery for thoracic aortic aneurysm were evaluated. The grafts anastomosed on the vascular prosthesis (group P, $n=75$ ) were compared to those anastomosed on the native ascending aorta (group N, $n=$ 34) by computed tomography angiography (CTA) and coronary angiography.

- After 45.9 39.7-month follow-up, significantly worse patency of the grafts in group P was revealed when compared with those in group N $(100 \%$ vs $77.6 \%$ in 12 months, $100 \%$ vs $52.7 \%$ in 36 months, and $100 \%$ vs $31.6 \%$ in 57 months, $P<0.001$ ).

- The poor patency of the grafts was confirmed in each target lesions (left anterior descending (LAD): $P=0.050$, right coronary artery (RCA): $P=0.045$, left circumflex (LCX): $P=0.051$ ) and regardless of the severities of the target coronary vessels (severe stenosis: $P=0.013$, mildto-moderate stenosis: $P=0.029$ ).

Comments Although concomitant surgery for coronary artery disease (CAD) and thoracic aortic aneurysm is performed often, the long-term patency of the CABG anastomosed to a vascular prosthesis has not been fully investigated. The cause of the CABG graft occlusion is multifactorial. Early graft failure is attributed to technical problems of the surgery and resulting thrombosis, while late graft failures are mainly caused by the progression of atherosclerosis on the graft or intimal hyperplasia at the anastomosis site. Authors have hypothesized that in their study, some mechanisms, that raised the intimal hyperplasia at the proximal anastomosis site on the vascular prosthesis, might have existed.

Take-home message In concomitant CABG and thoracic aorta surgery, proximal anastomosis of saphenous vein on vascular prosthesis should be avoided.

\section{Trifecta versus Perimount Magna Ease aortic valve prostheses}

Biancari F, Valtola A, Juvonen $\mathrm{T}$, et al. Trifecta versus Perimount Magna Ease aortic valve prostheses. Ann Thorac Surg. 2020;110:879-888. doi: https://doi.org/10.1016/j. athoracsur.2019.12.071.

\section{Key points}

- FinnValve registry data of patients undergoing aortic valve replacement (AVR) with bioprosthesis between 2008 and 2017 was analyzed.

- Outcomes of patients receiving Trifecta (851 patients) and Perimount Magna Ease (1365 patients) bioprostheses (mean age, $74.1 \pm 6.7$ years; age $<65$ years, $8.9 \%$; mean follow-up, $3.8 \pm 2.1$ years) were studied.

- The rates of late mortality and prosthetic valve endocarditis were comparable. Trifecta has significantly lower transvalvular gradients immediately after surgical AVR, but this advantage over the Perimount Magna Ease does not to persist at 1 year. After propensity score matching, at 7 years, the Trifecta cohort had a higher risk of repeat AVR for structural valve failure (5.7\% vs $0 \%, P=0.009)$. 
Comments It is the first study to compare mid-term results of two very commonly used bovine pericardial bioprostheses for surgical AVR (The Trifecta (Abbott, IL, USA) and the Perimount aortic valve bioprostheses (Edwards, Lifesciences Corp., Irvine, USA)). However, many earlier studies have reported a lesser reoperation rate with Trifecta valve. It is worth noting that the Trifecta is a bioprosthetic with a sheet of bovine pericardium externally mounted on a titanium stent. This is designed to optimize the valve opening area. Excessive pressure on the strut base, leading to weakening of the overlying pericardium, may be a mechanism for early Trifecta failure. This may frequently occur when too large a valve is forced into the annulus. During handtying of knots, bending of the strut posts may occur. Other than being a retrospective non-randomized study, this study has an important limitation of lack of detailed echocardiographic data. Still this study points to a concerning higher failure rate of Trifecta valve bioprosthesis, when compared with Perimount aortic valve bioprosthesis, for AVR.

Take-home message Perimount Magna Ease aortic valve bioprosthesis has an edge over Trifecta valve bioprosthesis in terms of mid-term structural valve degeneration.

\section{Transcatheter aortic valve implantation and its im- pact on mitral valve geometry and function}

Meyer A, Greve D, Unbehaun A, et al. Transcatheter aortic valve implantation and its impact on mitral valve geometry and function. J Card Surg. 2020; 35: 2185-2193. https://doi. org/10.1111/jocs. 14734

\section{Key points}

- 84 patients, who underwent Transcatheter aortic valve implantation (TAVI; balloon-expandable valve - 44; and self-expandable valve - 40), were evaluated for change in mitral valve (MV) geometry by $3 \mathrm{D}$ volumetric transesophageal echocardiography (TEE) before and immediately after TAVI using a dedicated software (4 D MV ASSESSMENT tool; TOMTEC imaging systems).

- During systole, the anterior to posterior (AP) diameter, mitral annular area, and tenting area were significantly reduced after the TAVI.

- Patients treated with balloon-expandable valves showed significantly larger reduction in the AP diameter, compared to self-expandable valves. Grade of mitral regurgitation (MR) improved, or remained stable, after TAVI.

Comments A significant number of patients undergoing TAVI have functional MR. Improvement in MR after TAVI is often attributed to reduced end-systolic pressure after TAVI. While changes in MV geometry have been well described after surgical AVR, this study demonstrates similar changes in MV geometry after TAVI which may also attribute to the reduction of MR after TAVI. As per this study, the reduction in AP diameter of MV is more with balloon-expandable valves, which may be relevant for planning procedures and device selection. Larger cohorts and long-term follow-up are needed to further comment on these observations in the study.

Take-home message TAVI significantly alters the MV geometry. Device selection (balloon vs self-expanding) for TAVI also should be done, considering their impact on MV geometry.

VI. Is myocardial revascularization really necessary in patients with $\geq \mathbf{5 0 \%}$ but $<\mathbf{7 0 \%}$ coronary stenosis undergoing valvular surgery?

Benedetto DF, Guido A, Elisabetta L, et al. Is myocardial revascularization really necessary in patients with $\geq 50 \%$ but $<70 \%$ coronary stenosis undergoing valvular surgery? Euro $J$ Cardiothorac Surg. 2020;58:343-349, https://doi.org/10. 1093/ejcts/ezaa047.

\section{Key points}

- 77 consecutive patients undergoing valvular heart surgery (VHS), in whom a de novo diagnosis of $\geq 50 \%$, but $<70 \%$ coronary stenosis was made, were studied. In this cohort, the myocardial revascularization was omitted. All these patients were free from angina and ischemia on echocardiography and electrocardiogram.

- No in-hospital deaths. In only 1 patient, acute MI occurred postoperatively, which was immediately treated by percutaneous intervention (PCI). The 6-year overall survival was $94.7 \pm 2.59 \%$. At 6 years, no cardiac deaths were recorded. At follow-up, 4 patients underwent elective PCI after a positive stress myocardial perfusion test. Only 1 patient underwent urgent PCI due to acute coronary syndrome. At 6 years, the cumulative incidence function of PCI, with death as competing risk, was $8 \pm 3.9 \%$.

Comments Three issues associated with grafting moderate stenosis coronaries are as follows: competitive flow due to intermediate coronary stenosis affects the patency rate of the graft; moderate coronary stenosis may be affected by early disease progression when bypassed; and moderate coronary stenosis with negative fractional flow reserve (FFR) may be left untreated and safely followed. Also, the latest European Society of Cardiology guidelines on valvular heart diseases have classified CABG at the time of VHS as class IIa (level of evidence C) for coronary stenosis $\geq 50-70 \%$. On the other hand, the American 
College of Cardiology guidelines have re-classified CABG at the time of AVR for $\geq 70 \%$ stenosis from a class I to a class IIa recommendation and de-emphasized the role of coronary revascularization in those with $50-70 \%$ coronary stenosis.

Take-home message Moderate coronary stenosis detected at the time of valve surgery can be safely overlooked and does not need any further treatment at follow-up in most cases.

Funding Nil.

\section{Compliance with ethical standards}

Ethics approval and consent to participate Not required as this section is purely for academic purposes and it fully acknowledges the original work by authors and publishers. Permission of copyright material has been sought where-ever necessary.

Conflict of interest The authors declare that they have no conflict of interest.

Publisher's note Springer Nature remains neutral with regard to jurisdictional claims in published maps and institutional affiliations. 